\title{
ASYMPTOTIC BEHAVIOUR OF FIRMLY NONEXPANSIVE SEQUENCES
}

\author{
BEHZAD DJAFARI ROUHANI
}

(Communicated by Palle E. T. Jorgenson)

\begin{abstract}
We introduce the notion of firmly nonexpansive sequences in a Banach space and present several results concerning their asymptotic behaviour extending previous results and giving an affirmative answer to an open question raised by S. Reich and I. Shafrir [Proc. Amer. Math. Soc. 101 (1987), 246-250]. Applications to averaged mappings are also given.
\end{abstract}

\section{INTRODUCTION}

Let $X$ be a real Banach space and $D$ a nonempty subset of $X$; the norms of both $X$ and $X^{*}$ are denoted by ||; we denote weak convergence in $X$ by $\rightarrow$ and strong convergence by $\rightarrow$. A mapping $T: D \rightarrow X$ is said to be firmly nonexpansive $[2,3]$ if for each $x$ and $y$ in $D$, the convex function $f:[0,1] \rightarrow[0, \infty[$ defined by $f(t)=|(1-t)(x-y)+t(T x-T y)|$ is nonincreasing. Equivalently, $T$ is firmly nonexpansive if and only if $|T x-T y| \leq$ $|t(x-y)+(1-t)(T x-T y)|$ for all $x, y \in D$ and $t \in[0,1]$, or, if and only if $T$ is the resolvent $(I+A)^{-1}$ for some accretive operator $A \subset X \times X$. Note also that any linear projection of norm one is firmly nonexpansive. Properties of such mappings, as well as averaged mappings, were studied by Reich [15], Bruck and Reich [4], Baillon, Bruck, and Reich [1]; and their results were extended by Plant and Reich [14] and Reich and Shafrir [16]. We refer also to the two textbooks on the subject by Goebel and Reich [11] and Goebel and Kirk [10] without recalling the basic concepts.

In this paper we introduce the notion of firmly nonexpansive sequences in a Banach space, and by using our previous methods in $[5,6]$ for bounded sequences and in $[7,8]$ for unbounded sequences we study the asymptotic behaviour of such sequences, extending the previous results mentioned above and, in particular, giving an affirmative answer to an open question raised by Reich and Shafrir [16, p. 249]. Applications to averaged mappings are also given, extending previous results in $[1,16]$.

\section{Preliminaries}

(a) The sequence $\left(x_{n}\right)_{n \geq 0}$ in $X$ is nonexpansive if $\left|x_{i+1}-x_{j+1}\right| \leq\left|x_{i}-x_{j}\right|$ for all $i, j \geq 0$.

Received by the editors May 28, 1993; presented at the ICTP, Trieste, Italy, in May 1993.

1991 Mathematics Subject Classification. Primary 47H09. 
(b) $\left(x_{n}\right)_{n \geq 0}$ is a firmly nonexpansive sequence (abbreviated F.N.E.S.) if the function $f:[0,1] \rightarrow\left[0, \infty\left[\right.\right.$ defined by $f(t)=\left|(1-t)\left(x_{i}-x_{j}\right)+t\left(x_{i+1}-x_{j+1}\right)\right|$ is nonincreasing for all $i, j \geq 0$.

(c) $\left(x_{n}\right)_{n \geq 0}$ is an odd firmly nonexpansive sequence (abbreviated O.F.N.E.S.) if it is a F.N.E.S. and $\left|x_{i+1}+x_{j+1}\right| \leq\left|x_{i}+x_{j}\right|$ for all $i, j \geq 0$.

(d) $\left(x_{n}\right)_{n \geq 0}$ is asymptotically regular (resp. weakly asymptotically regular) if $x_{n+1}-x_{n} \rightarrow_{n \rightarrow \infty} 0$ (resp. $x_{n+1}-x_{n} \rightarrow_{n \rightarrow \infty} 0$ ).

(e) If $D$ is a nonempty subset of $X$, we denote by clco $D$ the closed convex hull of $D$ in $X$ and, for $x \in X, d(x, D)=\inf _{z \in D}|x-z|$. We denote by $\bar{D}$ the strong closure of $D$ in $X$. We say that $D$ has the minimum property if $d(0, D)=d(0$, clco $D)$. For an operator (possibly multivalued) $A \subset X \times X$, $R(A)$ will denote the range of $A$.

(f) For a sequence $\left(x_{n}\right)_{n \geq 0}$ in $X$, we denote by $F\left(\left(x_{n}\right)_{n \geq 0}\right)$ or for simplicity by $F$ the following subset (possibly empty) of $X: F=\left\{q \in X / \lim _{n \rightarrow+\infty}\left|x_{n}-q\right|\right.$ exists $\}$. Note that if $F \neq \varnothing$, then the sequence $\left(x_{n}\right)_{n \geq 0}$ is bounded. Similarly we denote $F_{1}=\left\{q \in X /\right.$ the sequence $\left(\left|x_{n}-q\right|\right)_{n \geq 0}$ is nonincreasing $\} \subset F$.

(g) Given a bounded sequence $\left(x_{n}\right)_{n \geq 0}$ in $X$ and a nonempty closed subset $K$ of $X$, the asymptotic radius of $\left(x_{n}\right)_{n \geq 0}$ in $K$ is the number $r\left(K,\left(x_{n}\right)_{n \geq 0}\right)=$ $\inf _{x \in K}\left(\limsup \sup _{n \rightarrow+\infty}\left|x_{n}-x\right|\right)$. The asymptotic centre of $\left(x_{n}\right)_{n \geq 0}$ in $K$ is the (possible empty) set defined by

$$
A\left(K,\left(x_{n}\right)_{n \geq 0}\right)=\left\{x \in K / \limsup _{n \rightarrow+\infty}\left|x_{n}-x\right|=r\left(K,\left(x_{n}\right)\right)_{n \geq 0}\right\} .
$$

If $K$ is weakly compact, then $A\left(K,\left(x_{n}\right)_{n \geq 0}\right) \neq \varnothing$; if $K$ is convex, then $A\left(K,\left(x_{n}\right)_{n \geq 0}\right)$ is convex. In a Hilbert space $H$, for $K \supset \operatorname{clco}\left\{\left(x_{n}\right)_{n \geq 0}\right\}$, $A\left(K,\left(x_{n}\right)_{n \geq 0}\right)$ is a singleton and independent of $K$ (see $\left.[10,11]\right)$.

(h) We recall that if $X$ is reflexive and strictly convex and $K$ a nonempty closed convex subset of $X$, the nearest point projection map $P_{K}$ of $X$ onto $K$ is well defined, i.e., $K$ is a Chebyshev set; see $[10,11]$.

(i) The norm of $X$ is Fréchet differentiable if for each $x \in S=\{z \in X /|z|=$ 1\}, $\lim _{t \rightarrow 0} \frac{|x+t y|-|x|}{t}$ exists uniformly for $y \in S$. We recall also that the dual space $X^{*}$ has Fréchet differentiable norm if and only if $X$ is reflexive, strictly convex, and satisfies the following property: if $x_{n} \rightarrow_{n \rightarrow \infty} x$ and $\left|x_{n}\right| \rightarrow_{n \rightarrow \infty}|x|$, then $\left|x_{n}-x\right| \rightarrow_{n \rightarrow \infty} 0$ (see [9]).

(j) Throughout the paper we will use the following notation:

$$
C=\operatorname{clco}\left\{\left(x_{n+1}-x_{n}\right)_{n \geq 0}\right\} .
$$

\section{SOME BASIC RESULTS}

First we recall the following theorem, which is the same as Theorem 3.1 in [8], thus omitting its proof.

Theorem 3.1. Let $\left(x_{n}\right)_{n \geq 0}$ be a nonexpansive sequence in $X$. Then

$$
\lim _{n \rightarrow+\infty}\left|\frac{x_{n}}{n}\right|=\inf _{n \geq 1}\left|\frac{x_{n}-x_{0}}{n}\right|=d(0, C) .
$$

Now we prove some additional results which will be used in the sequel. 
Proposition 3.2. Let $\left(x_{n}\right)_{n \geq 0}$ be a nonexpansive sequence in $X$. Then

Proof. We have

$$
\begin{aligned}
\lim _{n \rightarrow+\infty}\left|\frac{x_{n}}{n}\right| & =d(0, C)=\lim _{k \rightarrow \infty} \lim _{n \rightarrow \infty}\left|\frac{x_{n+k}-x_{n}}{k}\right| \\
& =\inf _{k \geq 1} \lim _{n \rightarrow \infty}\left|\frac{x_{n+k}-x_{n}}{k}\right| \\
& =\inf _{k \geq 1} \lim _{n \geq 1}\left|\frac{x_{n+k}-x_{n}}{k}\right| .
\end{aligned}
$$

$$
\frac{x_{n+k}-x_{n}}{k}=\frac{1}{k} \sum_{i=1}^{k}\left(x_{n+i}-x_{n+i-1}\right) \in C .
$$

Hence $\forall n \geq 0, \forall k \geq 1$,

$$
d(0, C) \leq\left|\frac{x_{n+k}-x_{n}}{k}\right| \leq\left|\frac{x_{k}-x_{0}}{k}\right| .
$$

Now all the above equalities follow from Theorem 3.1.

Our next theorem extends Theorem 1 in [16].

Theorem 3.3. If $\left(x_{n}\right)_{n \geq 0}$ is a F.N.E.S. in $X$, then in addition to the conclusions of Theorem 3.1 and Proposition 3.2 we have: $\forall k \geq 1$,

$$
\lim _{n \rightarrow+\infty}\left|x_{n+1}-x_{n}\right|=\lim _{n \rightarrow+\infty}\left|\frac{x_{n+k}-x_{n}}{k}\right|=\lim _{n \rightarrow+\infty}\left|\frac{x_{n}}{n}\right|=d(0, C) .
$$

Proof. Let $R_{k}=\lim _{n \rightarrow+\infty}\left|x_{n+k}-x_{n}\right|$ for $k \geq 1$. We have $\forall k \geq 1$,

$$
\left|\frac{x_{n+k}-x_{n}}{k}\right| \leq \frac{1}{k} \sum_{i=1}^{k}\left|x_{n+i}-x_{n+i-1}\right| \rightarrow_{n \rightarrow+\infty} \lim _{n \rightarrow+\infty}\left|x_{n+1}-x_{n}\right|=R_{1} .
$$

Hence $R_{k} \leq k R_{1}$ for all $k \geq 1$. To complete the proof, by Proposition 3.2, all we need to show is the converse inequality $R_{k} \geq k R_{1}$ for all $k \geq 1$. The proof is similar to that of Theorem 1 in [16] and we use induction on $k$. Since the case $k=1$ is clear, we assume that $R_{j}=j R_{1}$ for all $1 \leq j \leq k$ and prove that $R_{k+1} \geq(k+1) R_{1}$.

Given $\varepsilon>0$, we can find an integer $N(\varepsilon)$ such that $R_{1} \leq\left|x_{n+j}-x_{n}\right| / j \leq$ $R_{1}+\varepsilon$ for all $1 \leq j \leq k$ and $n \geq N(\varepsilon)$. But since $\left(x_{n}\right)_{n \geq 0}$ is a F.N.E.S., we have

$$
\begin{aligned}
\left|x_{n+1}-x_{n+k+1}\right| & \leq \frac{1}{2}\left|\left(x_{n}-x_{n+k}\right)+\left(x_{n+1}-x_{n+k+1}\right)\right| \\
& \leq \frac{1}{2}\left|x_{n}-x_{n+k+1}\right|+\frac{1}{2}\left|x_{n+1}-x_{n+k}\right| .
\end{aligned}
$$

Hence,

$$
\begin{aligned}
\left|x_{n}-x_{n+k+1}\right| & \geq 2\left|x_{n+1}-x_{n+k+1}\right|-\left|x_{n+1}-x_{n+k}\right| \\
& \geq 2 k R_{1}-(k-1)\left(R_{1}+\varepsilon\right)=(k+1) R_{1}-(k-1) \varepsilon
\end{aligned}
$$

for all $n \geq N(\varepsilon)$.

Therefore, $R_{k+1} \geq(k+1) R_{1}$ since $\varepsilon>0$ is arbitrary, and the proof of the theorem is now complete. 


\section{ASYMPTOTIC BEHAVIOUR OF FIRMLY NONEXPANSIVE SEQUENCES}

We have the following theorem concerning the convergence of the sequence $y_{n}=x_{n+1}-x_{n}$ which extends Theorem 2 in [16] and gives an affirmative answer to an open question posed in [16, p. 249] concerning the sufficiency of the Fréchet differentiability of the norm of $X^{*}$ for the convergence of $y_{n}$.

Theorem 4.1. Let $\left(x_{n}\right)_{n \geq 0}$ be a F.N.E.S. in $X$ and $y_{n}=x_{n+1}-x_{n}$.

(i) If $X$ is reflexive and strictly convex, then $y_{n} \rightarrow_{n \rightarrow+\infty} P_{C} 0$. Moreover, $\lim _{n \rightarrow+\infty}\left|y_{n}\right|=\left|P_{C} 0\right|$.

(ii) If $X^{*}$ has Fréchet differentiable norm, then $y_{n} \rightarrow_{n \rightarrow+\infty} P_{C} 0$.

Proof. $X$ being reflexive, $y_{n}$ contains a weakly convergent subsequence; let $y_{n_{k}} \rightarrow_{k \rightarrow \infty} p \in C . X$ being also strictly convex, we have $d(0, C)=\left|P_{C} 0\right|$. Therefore, $|p| \leq \liminf _{k \rightarrow+\infty}\left|y_{n_{k}}\right|=\lim _{n \rightarrow+\infty}\left|y_{n}\right|=\left|P_{C} 0\right|$ by Theorem 3.3. Hence, we must have $p=P_{C} 0$; this shows that $y_{n}$ converges weakly to $P_{C} 0$ with $\lim _{n \rightarrow+\infty}\left|y_{n}\right|=\left|P_{C} 0\right|$ and completes the proof of (i).

Now (ii) is an immediate consequence of (i) and the characterization of $X$ given in $\S 2(\mathrm{i})$.

Remark 4.2. The example of [13] can be used to show that the assumptions made on $X$ in (i) or (ii) are also necessary for the respective conclusion to hold.

Corollary 4.3. Let $\left(x_{n}\right)_{n \geq 0}$ be a F.N.E.S. in $X$. Then the following are equivalent:

(i) $\left(x_{n}\right)_{n \geq 0}$ is asymptotically regular.

(ii) $\left(x_{n}\right)_{n \geq 0}$ is weakly asymptotically regular.

(iii) $y_{n}=x_{n+1}-x_{n}$ has a weakly convergent subsequence to zero.

Proof. Assume $y_{n_{k}}=x_{n_{k}+1}-x_{n_{k}} \rightarrow_{k \rightarrow \infty} 0$. Then $0 \in C$; hence, $d(0, C)=$ $0=\lim _{n \rightarrow+\infty}\left|x_{n+1}-x_{n}\right|$ by Theorem 3.3 . Therefore, $\left(x_{n}\right)_{n \geq 0}$ is asymptotically regular, and the proof is complete.

Corollary 4.4. Let $\left(x_{n}\right)_{n \geq 0}$ be a F.N.E.S. in $X$. Then $\left(x_{n}\right)_{n \geq 0}$ is asymptotically regular if $\lim _{\inf } \operatorname{lo}_{n \rightarrow+\infty}\left|x_{n}\right|<+\infty$.

Proof. This follows directly from Theorem 3.3 since in this case $\lim _{n \rightarrow+\infty}\left|x_{n} / n\right|$ $=0$.

Example 4.5. The following example shows that the converse does not hold in Corollary 4.4 even in one dimension. Let $x_{0}=0$ and $x_{n}=\sum_{k=1}^{a} \frac{1}{k}$ for $n \geq 1$.

Then it is easily verified that $\left(x_{n}\right)_{n \geq 0}$ is a F.N.E.S. of real numbers satisfying $x_{n} \rightarrow_{n \rightarrow+\infty}+\infty$ and $x_{n+1}-x_{n}=\frac{1}{n+1} \rightarrow_{n \rightarrow+\infty} 0$.

Now we study the convergence of $x_{n}$ in a Hilbert space. Our next theorem extends (for the case of a Hilbert space) Corollary 4 in [16].

Theorem 4.6. Let $\left(x_{n}\right)_{n \geq 0}$ be a F.N.E.S. in a real Hilbert space $H$. Then the following are equivalent:

(i) $\left|x_{n}\right|$ is bounded.

(ii) $\liminf _{n \rightarrow+\infty}\left|x_{n}\right|<+\infty$.

(iii) $F_{1} \neq \varnothing$

(iv) $F \neq \varnothing$.

(v) $x_{n}$ converges weakly in $H$. 
Moreover, the weak limit in (v) can be identified as $\lim _{n \rightarrow+\infty} P_{F_{1}} x_{n}$ and also as the asymptotic center of the sequence $\left(x_{n}\right)_{n \geq 0}$.

Proof. We note that by Corollary $4.4\left(x_{n}\right)_{n \geq 0}$ is asymptotically regular. Hence, the proof is completed by applying Proposition 2.10 and Theorem 2.11 in [5] and Theorem 3.4 and Corollary 3.10 in [6].

Remark 4.7. Example 3.5 in [6] shows that the sequence $P_{F} x_{n}$ may not be convergent in $H$.

Remark 4.8. The conclusions of Theorem 4.6 seem to be still open problems in a Banach space setting.

Now we give some conditions for the strong convergence of a F.N.E.S. in a Banach space. Our next theorem is simple.

Theorem 4.9. Let $\left(x_{n}\right)_{n \geq 0}$ be a F.N.E.S. in $X$ satisfying $\left|x_{i+m}-x_{i}\right| \rightarrow_{i \rightarrow+\infty} \alpha_{m}$ uniformly in $m \geq 0$. Then $x_{n}$ converges strongly to some $p \in F_{1}$ if and only if $\liminf _{n \rightarrow+\infty}\left|x_{n}\right|<+\infty$.

Proof. By Corollary 4.4 we have $\alpha_{1}=0$; hence, by Theorem 3.3, $\alpha_{m}=m \alpha_{1}=$ 0 for all $m \geq 1$. This shows that $\left(x_{n}\right)_{n \geq 0}$ is a Cauchy sequence in $X$ and, hence, strongly convergent to some $p \in X$. We have $\left|x_{i+m+1}-x_{i+1}\right| \leq\left|x_{i+m}-x_{i}\right|$ for all $i, m \geq 0$; letting $m \rightarrow+\infty$ we get $\left|x_{i+1}-p\right| \leq\left|x_{i}-p\right|$ for all $i \geq 0$. Thus $p \in F_{1}$ and the proof is complete.

Remark 4.10. Obviously the strong limit of any sequence is its asymptotic centre with respect to any set containing the limit.

Our next theorem is similar to Theorem 1.1 in [1].

Theorem 4.11. Let $\left(x_{n}\right)_{n \geq 0}$ be an O.F.N.E.S. in a uniformly convex Banach space $X$. Then $x_{n}$ converges strongly as $n \rightarrow+\infty$ to some $p \in F_{1}$.

Proof. The oddness implies that $\lim _{n \rightarrow+\infty}\left|x_{n}\right|=d$ exists, and hence, by Corollary $4.4\left(x_{n}\right)_{n \geq 0}$ is asymptotically regular. Now we have $2 d \leq 2\left|x_{n}\right| \leq$ $\left|x_{n}+x_{n+m}\right|+\left|x_{n}-x_{n+m}\right|$ for all $n, m \geq 0$. But for fixed $m \geq 0$, we have $\left|x_{n}-x_{n+m}\right| \rightarrow_{n \rightarrow+\infty} 0$, hence, $2 d \leq \lim _{n \rightarrow+\infty}\left|x_{n}+x_{n+m}\right|$ for all $m \geq 0$, and therefore $2 d \leq\left|x_{n}+x_{n+m}\right| \leq\left|x_{n}\right|+\left|x_{n+m}\right|$ for all $n, m \geq 0$. This implies that $\lim _{n \rightarrow+\infty}\left|x_{n}+x_{n+m}\right|=2 d$ uniformly in $m \geq 0$. By uniform convexity, we deduce that $\lim _{n \rightarrow+\infty}\left|x_{n}-x_{n+m}\right|=0$ uniformly in $m \geq 0$; hence, $\left(x_{n}\right)_{n \geq 0}$ is a Cauchy sequence in $X$, thus strongly convergent. The same argument as in Theorem 4.9 shows that the limit belongs to $F_{1}$ and completes the proof.

\section{ASYMPTOTIC BEHAVIOUR OF AVERAGED MAPPINGS}

Let $K$ be a nonempty closed convex subset of $X$ and $T: K \rightarrow K$ a nonexpansive mapping (i.e., $|T x-T y| \leq|x-y|$ for all $x, y \in K)$. By an averaged mapping $U: K \rightarrow K$ we mean a mapping of the form $U=(1-\lambda) I+\lambda T$ where $0<\lambda<1$ and $I$ is the identity. It is clear that $U$ is nonexpansive and has the same fixed-point set as $T$ and that $U$ is odd if and only if $T$ is odd. Ishikawa [12] has shown that $U$ is asymptotically regular if $K$ is bounded. Combining Theorem 3.1 with Theorem 2.1 in [1] and Corollary 2 in [16] and denoting $C_{x}=\operatorname{clco}\left\{\left(U^{n+1} x-U^{n} x\right)_{n \geq 0}\right\}$ for $x \in K$, we get the following theorem. 
Theorem 5.1. For all $k \geq 1$ and $x \in K$ we have

$$
\begin{aligned}
\lim _{n \rightarrow+\infty}\left|U^{n+1} x-U^{n} x\right| & =\frac{1}{k} \lim _{n \rightarrow+\infty}\left|U^{n+k} x-U^{n} x\right|=\lim _{n \rightarrow+\infty}\left|\frac{U^{n} x}{n}\right| \\
& =d\left(0, C_{x}\right)=\inf _{y \in K}|y-U y|=\lambda \inf _{y \in K}|y-T y| .
\end{aligned}
$$

Now the same argument as in $\S 4$ gives the following theorem for averaged mappings which extends Corollary 2.3 in [1] and its extensions made in [16, $\mathrm{p}$. 249].

Theorem 5.2. Let $U: K \rightarrow K$ be an averaged mapping and $x \in K$.

(i) If $X$ is reflexive and strictly convex, then $U^{n+1} x-U^{n} x \rightarrow_{n \rightarrow+\infty} P_{C_{x}} 0$. Moreover $\lim _{n \rightarrow+\infty}\left|U^{n+1} x-U^{n} x\right|=\left|P_{C_{x}} 0\right|$ and $P_{C_{x}} 0$ is independent of $x \in K$. If in addition $\overline{R(I-T)}$ has the minimum property, then $-\frac{1}{\lambda} P_{C_{x}} 0$ is the element of minimum norm in $\overline{R(I-T)}$.

(ii) If $X^{*}$ has Fréchet differentiable norm, then $U^{n+1} x-U^{n} x \rightarrow_{n \rightarrow+\infty} P_{C_{x}} 0$ and $-\frac{1}{\lambda} P_{C_{x}} 0$ is the element of minimum norm in $\overline{R(I-T)}$.

Proof. It is similar to that of Theorem 4.1 by using Theorem 5.1. Concerning the statements about $\overline{R(I-T)}$, it is clear in (i), and in (ii) the argument is similar to Theorem 2 in [16] using the fact that $(I-T)$ is accretive. We therefore omit the details.

Corollary 5.3. Let $U: K \rightarrow K$ be an averaged mapping and $x \in K$. Then the following are equivalent:

(i) $U$ is asymptotically regular at $x \in K$.

(ii) $U$ is weakly asymptotically regular at $x \in K$.

(iii) The sequence $\left(U^{n+1} x-U^{n} x\right)_{n \geq 0}$ has a weakly convergent subsequence to zero.

Proof. It is similar to that of Corollary 4.3 by using Theorem 5.1 .

\section{ACKNOWLEDGMENTS}

The author thanks Professor Abdus Salam, the International Atomic Energy Agency, and UNESCO for hospitality at the International Centre for Theoretical Physics, Trieste.

\section{REFERENCES}

1. J. B. Baillon, R. E. Bruck, and S. Reich, On the asymptotic behaviour of nonexpansive mappings and semigroups in Banach spaces, Houston J. Math. 4 (1978), 1-9.

2. F. E. Browder, Convergence theorems for sequences of nonlinear operators in Banach spaces, Math. Z. 100 (1967), 201-225.

3. R. E. Bruck, Nonexpansive projections on subsets of Banach spaces, Pacific J. Math. 47 (1973), 341-355.

4. R. E. Bruck and S. Reich, Nonexpansive projections and resolvents of accretive operators in Banach spaces, Houston J. Math. 3 (1977), 459-470.

5. B. Djafari Rouhani, Asymptotic behaviour of quasi-autonomous dissipative systems in Hilbert spaces, J. Math. Anal. Appl. 147 (1990), 465-476.

6. _ Asymptotic behaviour of almost nonexpansive sequences in a Hilbert space, J. Math. Anal. Appl. 151 (1990), 226-235. 
7. _ A simple proof to an extension of a theorem of A. Pazy in Hilbert space, preprint, ICTP, Trieste, No. IC/90/219, 1990.

8. __ Asymptotic behaviour of unbounded nonexpansive sequences in Banach spaces, Proc. Amer. Math. Soc. 117 (1993), 951-956.

9. K. Fan and I. Glicksberg, Some geometric properties of the spheres in a normed linear space, Duke Math J. 25 (1958), 553-568.

10. K. Goebel and W. A. Kirk, Topics in metric fixed point theory, Cambridge Stud. in Adv. Math., vol. 28, Cambridge, New York, Melbourne, and Sydney, 1990.

11. K. Gcebel and S. Reich, Uniform convexity, hyperbolic geometry and nonexpansive mappings, Dekker, New York and Basel, 1984.

12. S. Ishikawa, Fixed points and iteration of a nonexpansive mapping in a Banach space, Proc. Amer. Math. Soc. 59 (1976), 65-71.

13. E. Kohlberg and A. Neyman, Asymptotic behaviour of nonexpansive mappings in normed linear spaces, Israel J. Math. 38 (1981), 269-275.

14. A. T. Plant and S. Reich, The asymptotics of nonexpansive iterations, J. Funct. Anal. 54 (1983), 308-319.

15. S. Reich, On the asymptotic behaviour of nonlinear semigroups and the range of accretive operators, I, II, Mathematics Research Center Report 2198 (1981); J. Math. Anal. Appl. 79 (1981), 113-126; 87 (1982), 134-146.

16. S. Reich and I. Shafrir, The asymptotic behaviour of firmly nonexpansive mappings, Proc. Amer. Math. Soc. 101 (1987), 246-250.

International Centre for Theoretical Physics, Trieste, Italy

Current address: Postgraduate and Research Branch, Islamic Azad University, and School of Mathematical Sciences, Shahid Beheshti University, Tehran, Iran 